\title{
Dynamic Ball Indentation for powder flow characterization
}

\author{
Monica Tirapelle ${ }^{\mathrm{a}, *}$, Andrea C. Santomaso ${ }^{\mathrm{a}, * *}$, Colin Hare $^{\mathrm{b}}$ \\ ${ }^{a}$ APTLab - Advanced Particle Technology Laboratory, Department of Industrial \\ Engineering, University of Padova, IT \\ ${ }^{b}$ Department of Chemical and Process Engineering, University of Surrey, Guildford, \\ GU27XH, UK
}

\begin{abstract}
In industrial processing and manufacturing, characterizing the flowability of particulate solids is of particular importance both for reliable powder flow and for a consistent production rate. Shear testing is the most widely used method for powders subjected to moderate or high stresses, and under quasi-static conditions. However, this method is not suitable for measuring the powder flow properties occurring in dynamic systems, such as powder mixers and screw conveyors. In this study, the rheological behaviour of powders at high shear rates has been evaluated by the ball indentation method. The technique, which simply consists of dropping a ball onto a cylindrical bed of previously consolidated powder, directly measures the material hardness, which is related to the unconfined yield stress by the constraint factor. The impact of the ball on the bed is recorded with a high-speed camera to determine velocity and penetration depth. The hardness against the strain rate is considered for four different materials. Because of their difference in particle size, and by using a range of drop heights and a range of indenter densities, the intermediate regime of flow has been fully analyzed. Although hardness is constant in the quasi-static condition, it results to be strain rate dependent in the intermediate regime of flow. Finally,
\end{abstract}

\footnotetext{
* Corresponding author. Email address : monica.tirapelle@phd.unipd.it

** Principal corresponding author.

Email address : andrea.santomaso@unipd.it
}

Preprint submitted to Journal of ${ }^{A} T_{E} X$ Templates

December 4, 2019 
a predictive correlation that allows the operator to choose the best operating conditions for achieving the desired flow regime is proposed, and the unconfined yield strength of the materials is inferred.

Keywords: powder flowability, powder rheology, dynamic flow, intermediate flow regime, ball indentation

\begin{tabular}{|c|c|c|c|}
\hline \multicolumn{4}{|c|}{ Nomenclature } \\
\hline$\dot{\gamma}$ & Shear rate $\left[s^{-1}\right]$ & $h *$ & Dimensionless \\
\hline$\dot{\gamma}^{*}$ & Dimensionless shear rate [-] & & depths $[-]$ \\
\hline$\rho_{b}$ & Powder bulk density $\left[\mathrm{kg} / \mathrm{m}^{3}\right]$ & $h_{0}$ & Penetration depth after un- \\
\hline$\rho_{i}$ & Indenter density $\left[\mathrm{kg} / \mathrm{m}^{3}\right]$ & & loading $[m]$ \\
\hline \multirow{2}{*}{$A$} & Projected area of the pene- & $H_{d}$ & Dynamic hardness $[k P a]$ \\
\hline & tration $\left[m^{2}\right]$ & $H_{s}$ & Quasi-static hardness $[k P a]$ \\
\hline$C$ & Constraint factor [-] & $h_{d r o p}$ & Drop height $[m]$ \\
\hline$d_{p}$ & Particle size $[m]$ & $M$ & Indenter mass $[k g]$ \\
\hline$F_{\max }$ & Maximum loading force $[N]$ & $R_{c}$ & Crater radius $[m]$ \\
\hline \multirow[t]{2}{*}{$g$} & Gravitational acceleration & $R_{i}$ & Indenter radius $[m]$ \\
\hline & {$\left[m / s^{2}\right]$} & $U$ & Unrelaxed volume $\left[\mathrm{m}^{3}\right]$ \\
\hline \multirow[t]{2}{*}{$h$} & Penetration depth at maxi- & $v_{i}$ & Impact velocity $[\mathrm{m} / \mathrm{s}]$ \\
\hline & $\operatorname{mum}$ force $[m]$ & $Y$ & Yield strength $[k P a]$ \\
\hline
\end{tabular}

\section{Introduction}

Granular materials are extensively used in industrial processing and manufacturing. Nevertheless their flow behaviour is still poorly understood. The reason is that a powder assembly can display a variety of flow properties at 
different stress states; it can manifest solid-like, liquid-like or gas-like behaviour [1, 2, 3. Granular material can also behave, under certain circumstances, in a completely different manner from that observed in all of the other states of matter [2, 4].

Because powder flow can be classified in different regimes, understanding the flow behaviour of powders as a function of the shear rate becomes of particular importance for both a reliable powder flow and a consistent production rate [5]. The conventional shear testers, such as the Jenike cell, annular cells, triaxial cells, and true biaxial testers, allow powder flow properties to be characterised only in the quasi-static regime [6, 7, 8, whereas, although there are a number of industrial cases where bulk solids are subject to high strain rates (e.g. highspeed bladed mixers, rotating mixers, screw conveyors) [3], only a few techniques allow the dynamic flow regime to be explored. These are the Couette device developed by Tardos et al. 9, which however is not commercially available, and the emergent powder rheometers $[9,10$. Although the latter could be useful for a comparative analysis of different powders, there is not yet a relationship that correlates the flow energy and the powder stress level. Here the dynamic ball indentation method is proposed as an alternative for capturing the rheological behaviour of powders subjected to high shear rates.

The ball indentation technique is a very common method used, for instance, 25 for evaluating hardness and tensile properties of continuum-solids, particularly for assessing the integrity of metallic structures [11, 12, 13], or for studying the process of formation and the morphology of planetary and lunar craters [14, 15, 16, 17. The same method has recently been proposed by Hassanpour and Ghadiri for evaluating the flowability of cohesive bulk powders at low compaction levels, in quasi-static conditions and at small scales [18]. The method, which consists of indenting the surface of a pre-consolidated powder bed with a ball, allows the material hardness, $H_{s}$, which is defined as the resistance of the material to plastic deformation, to be determined [19]. Although the tensile strength can not be measured directly by indentation, the hardness is related 35 to the unconfined yield stress through the constraint factor, $C$, that is, for bulk 
solids, distinctive of the material [20].

Here a distinction is made between quasi-static and dynamic indentation. With quasi-static indentation the indenter is driven at a constant rate, the penetration depth is controlled and the loading force is measured. Whereas with 40 the dynamic approach the indenter is released and accelerates under gravity. In this case the penetration depth cannot be controlled: it depends on the indenter kinetic energy and powder flow properties. This dynamic method has already been demonstrated through Discrete Element Method to be suitable for evaluating hardness of powder at high shear rates by Pasha et al. [5]. In particular they have investigated the stress-shear rate dependency of cohesive powders subjected to indentation in the intermediate and dynamic regimes of flow and have found that hardness, as well as deviatoric and hydrostatic stresses, are dependent on the indentation shear rate for dimensionless shear rates beyond unity [5].

The classification of the flow regimes is based on the dimensionless shear rate, $\dot{\gamma}^{*},($ Eq. 1) as proposed by Tardos et al. 9]:

$$
\dot{\gamma}^{*}=\dot{\gamma} \sqrt{\frac{d_{p}}{g}},
$$

where $d_{p}$ is the particle size, $g$ is the gravitational acceleration and $\dot{\gamma}$ is the shear rate. Specific to indentation, the shear rate $\dot{\gamma}$ is usually estimated with a simplified approach based on the impact velocity of the ball, $v_{i}$, and the indenter radius, $R_{i}\left(\dot{\gamma}=v_{i} / R_{i}\right)$ [5]. According to this classification, at zero shear rates the powder is in static condition. From the yield point, where the powder starts to displace, until dimensionless shear rates of $0.15-0.20$, the material is considered to be in the quasi-static or slow-frictional regime. Here, frictional interactions dominate. In the so called intermediate regime of flow, 60 for $0.25<\dot{\gamma}^{*}<3$, not only friction but also collisions between particles must be considered, with the combination from collisions increasing with shear rate. Finally, for dimensionless shear rates beyond 3, powder is in the dynamic regime of flow. Here, rapid and short duration collisions are predominant, individual powder particles are sufficiently energized and the powder assembly behaves like 
a freely flowing fluid. In this classification, the boundaries between regimes are still uncertain and not clearly defined [9, 2].

The present paper is concerned with the study of the rheological behaviour of bulk cohesive powders at high shear rates by experimentally applying the ball indentation method. To vary the shear rate, a range of indenter densities and

70 sizes, and a range of drop heights, are used. The hardness against the shear rate is considered for four different materials, whose constraint factor has been determined. This paper differs from a previous one [21] in that an improvement is made in the definition of the shear rate, $\dot{\gamma}$. Furthermore, the study has been extended on a fourth powder assembly for allowing the intermediate 75 regime of flow to be fully addressed. Finally a dimensionless number that scales all the measurements on the same master curve is defined, and hence allows any operator that wants to apply dynamic ball indentation to choose the most appropriate operating conditions.

\section{Experimental}

80 2.1. Materials

Four powder assemblies with different particle size, particle shape and flow behaviour have been tested. They are: Titanium Dioxide $\left(\mathrm{TiO}_{2}\right) \mathrm{R} 104$, Titanium Dioxide $\left(\mathrm{TiO}_{2}\right)$ DT51, $\alpha$-Lactose mono-hydrate and waxy Corn Starch.

In Table 1 some of their representative equivalent diameters are listed. It should be noted that they are all taken from volume distributions. The particle size of $\mathrm{TiO}_{2} \mathrm{R} 104$ and $\mathrm{TiO}_{2}$ DT51 was measured using the Malvern Zetasizer, with respectively $0.16 \mathrm{mg} / \mathrm{mL}$ and $0.29 \mathrm{mg} / \mathrm{mL}$ Butanone solutions. For lactose and corn starch, the size analysis was carried out in dry condition with the QicPic dynamic image analyzer. The data shows that $\mathrm{TiO}_{2} \mathrm{R} 104$ has the 90 smallest particle size, whilst lactose is the largest material tested, with median diameters respectively of $0.38 \mu \mathrm{m}$ and $82.80 \mu \mathrm{m}$. Since $\dot{\gamma}^{*}$ depends on particle diameter (see Eq. 1), this difference in size allows a wider range of dimensionless shear rates to be explored. 
The geometrical configuration and the surface structure of the materials

95 can be seen from the $S E M$ micrographs reported in Figure 1, both the grades of $\mathrm{TiO}_{2}$ exhibit a strong tendency to agglomerate (Fig. 1 fa and b). This is why, in order to disrupt the agglomerates, the size analysis have been carried out after sonicating the samples in an ultrasonic bath for 10 minutes. Furthermore, $\mathrm{TiO}_{2}$ DT51 has the most notable surface roughness. In terms of particle shape, all the powders are irregular with the exception of the starch. Note that the particles of $\alpha$-Lactose mono-hydrate are angular-shaped (Fig. 1 - $\mathrm{c}$ ) with sharp edges as a consequence of the milling process.

For characterizing the flow behaviour of the powders, the rotational shear tester of the FT4 Powder Rheometer (Freeman Technology, UK) was used. Figure 2 shows the material flow functions and Table 1 lists their obtained flow indexes, $f f$. According to the classification of powder flowability introduced by Jenike 22], $\mathrm{TiO}_{2} \mathrm{R} 104$ is classed as not flowing $(f f<1)$ at stresses lower than $20 \mathrm{kPa}, \mathrm{TiO}_{2}$ DT51 exhibits very cohesive $(1<f f<2)$ behaviour at lower stresses and cohesive $(2<f f<4)$ behaviour at higher stresses, whereas lactose and starch predominantly display easily flow $(4<f f<10)$ behaviour, with the former being cohesive under lower loads. It should be noted that, in order to investigate higher impact velocities, the powder must be more cohesive, since the higher kinetic energy of the indenter otherwise leads to unacceptably deep penetration. As expected, its clear that the cohesion decreases when particle size is increased.

\subsection{Method}

The methodologies provided by the FT4 powder rheometer (Freeman Technology) are used both for conditioning and consolidating the particulate solids.

The samples are firstly poured into a $50 \mathrm{~mL}$ cylindrical die with $50 \mathrm{~mm}$ diameter and then subjected to the pre-conditioning sequence of the rheometer. In such a way, any stress history is removed and the powder achieves a reproducible and homogeneous state. The conditioned powder beds are successively 
pre-consolidated to different stress levels by a stainless-steel vented piston that

moves downward with a velocity of $0.05 \mathrm{~mm} / \mathrm{s}$. Once the desired normal stress is reached, it is maintained for 1 minute to minimise elastic recovery. The consolidated bed is successively indented by applying ball indentation in quasi-static condition, or by performing the dynamic indentation process.

For evaluating the material hardness through the quasi-static ball indentation method, the rheometer is used. A $4 \mathrm{~mm}$ diameter indenter penetrates the sample at the constant speed of $0.05 \mathrm{~mm} / \mathrm{s}$ and the applied loading and unloading force are recorded as a function of the indentation depth. The hardness determination is therefore based on the loading force evaluated at the maximum indentation depth as proposed by Hassanpour and Ghadiri (Eq,2) [18, 19]:

$$
H_{s}=\frac{F_{\max }}{A}
$$

where $F_{\max }$ represents the maximum load applied and $A$ the projected area of the penetration [18. In turn $A$ is given by:

$$
A=2 \pi R_{i} h_{0}-\pi h_{0}^{2}
$$

The variables involved in Eq. 3, $h_{0}$ and $R_{i}$, are respectively the depth of the penetration after relaxation of the bed and the radius of the spherical indenter. However, since for all tests reported here the elastic recovery of the material has proved to be negligible (change in hardness value is less than $3 \%$ ), instead of $h_{0}$, the penetration depth at maximum force, $h$, has been used.

Measuring the quasi-static hardness of a powder at different controlled penetration depths is of particular importance because it allows the range of penetration depths where hardness measurements can be considered reliable to be determined. Indeed, as Fig. 3 shows, there exist a minimum and a maximum allowed penetration depth. Usually the minimum should be at least $20 \%$ the indenter radius to ensure the plastic deformation of the bed would be initialized, and the maximum should not be greater than $90 \%$ of the indenter radius. However, these limits are not well defined.

The dynamic hardness measurements are instead done with a customized 
device whose configuration requires a vacuum pump and a high-speed camera (see Fig. 4). The powder is firstly conditioned and pre-consolidated at the desire compression level by the rheometer. Then the indenter, which is initially held by the pump above the sample at a controlled variable height, is released by switching off the pump. The use of the pump guarantees the repeatability of the test and the consistency of the results. By placing the indenter at different heights, different impact velocities are investigated. The impact of the ball on the bed is recorded with a Phantom v1612 high-speed camera. By post processing image analysis of the frames, the radius of the crater, $R_{c}$, and the impact velocity of the sphere, $v_{i}$, are determined.

Sixteen different types of indenter, which differ for size and density, are employed here. They are high precision spherical balls made of Nylon, Glass, Ceramic and Stainless Steel, having the following diameters: 3.17, 4.77, 9.52 and $15.87 \mathrm{~mm}$. Unlike the quasi-static indentation procedure, the dynamic one does 165 not allow the loading/unloading curve nor the maximum indentation load, $F_{\max }$, to be recorded. Therefore, the determination of the dynamic hardness, $H_{d}$, is based on the kinetic energy as suggested by Tirupataiah and Sundararajan [23]:

$$
H_{d}=\frac{M v_{i}^{2}}{2 U},
$$

where $M$ is the mass of the indenter, $v_{i}$ is the impact velocity of the ball, and the crater volume, or unrelaxed volume, $U$ is calculated using the standard 170 geometrical relation for the volume of a spherical cap (Eq. 5).

$$
U=\frac{h \pi}{6}\left(3 R_{c}^{2}+h^{2}\right) .
$$

It is noteworthy that the size of samples and indenters, as well as the distance between the indentation zone and the surrounding walls, should be in accordance with the specifications of the operation window analyzed by Pasha et al. [20] and Zafar et al. 24. The criteria of the former have been adhered to here. 


\section{Results and discussion}

All the results reported in this section were obtained by performing the experiments under ambient conditions of $20-25^{\circ} \mathrm{C}$ and relative humidities of 45-60 \%. The error bars represent the standard deviation of three independent measurements.

\subsection{Quasi-static hardness}

To have reliable hardness measurements, the range of reliable penetration depths (see Fig. 3) should be necessarily identified. For this reason, before applying dynamic indentation, the quasi-static hardness needs to be measured for a wide range of dimensionless penetration depths $\left(h^{*} \equiv h / R_{i}\right)$.

Fig. 5 $\mathrm{a}$ and 5 b show the hardness measurements obtained by performing the indentation procedure provided by the rheometer for $h^{*}$ ranging from 0.1 to 0.9. The considered values of pre-compaction are different because each material tested was subjected to the same compression to which it was subjected in the case of dynamic hardness. The hardness of $\mathrm{TiO}_{2}$ R-104 is fairly stable for $h^{*}$ below 0.5 , whereas it increases for higher values of $h^{*}$. Therefore, hardness will be overestimated when $h^{*}$ is greater than 0.5. For Waxy Corn Starch and $\alpha$-Lactose mono-hydrate, slightly higher values are recorded only for $h^{*}$ respectively below 0.4 and 0.2 where, since the indenter is in contact with only a few particles, the bulk plastic deformation of the bed is not fully provided [24].

The existence of an initial non-linear regime was experimentally observed also by Kang et al.25. Indeed, by performing a range of penetration experiments with cylindrical intruders, they found that for $h^{*}<0.15$ the powder below the intruder is not in a condition of steady state consolidation; meanwhile for $h^{*}>$ 0.15 the formation of a stable cone of static material below the intruder occurs and steady state shearing conditions begin while the bed is plastically deformed 25]. Unlike for $\mathrm{TiO}_{2}$ R-104, Waxy Corn Starch and $\alpha$-Lactose mono-hydrate; $\mathrm{TiO}_{2}$ DT51 lacks a range of reliable penetration depths where hardness can be considered constant: hardness increases steadily with $h^{*}$. This is expected 
to impact negatively on the estimation of the dynamic hardness and also of

strength, and this powder is therefore not considered further.

Note that the variability with the material, and the possibility of no reliable range of penetration depths for indentation, are main drawbacks of this method.

\subsection{Dynamic ball indentation results}

Here the shear rate is defined as $\dot{\gamma}=v_{i} / R_{c}$. This definition is different from the one previously proposed in [5] and [26] where $\dot{\gamma}=v_{i} / R_{i}$. The crater radius, $R_{c}$, is indeed a more relevant physical property than $R_{i}$ since it is directly related to the penetration level of the spherical intruder into the powder and therefore is proportional to the amount of powder displaced by shearing. On the other and, $R_{i}$ is, for a given intruder, constant and completely independent of the penetration level.

Figure 6 6 a shows $\mathrm{H}_{d}$ of samples of $\mathrm{TiO}_{2}$ R104 pre-compacted to $20 \mathrm{kPa}$ as a function of the dimensionless shear rate. These results were achieved by impacting all the sixteen indenters at $1.99 \pm 0.04 \mathrm{~m} / \mathrm{s}$. It appears that hardness

220 is independent of the shear rate both in quasi-static regime and in the uncertain boundary below the intermediate regime. Although the measurements proved to be independent on the indenter type, Stainless Steel intruders overestimate hardness. The reason is that Stainless Steel, which is the heaviest material, penetrates deeper in the powder bed, reaching the range of penetration depths where hardness cannot be considered constant anymore, i.e. greater than 0.5 (see Fig. 5-a and Fig. 6-b). Along with the dynamic indentation results, in Figure 6-b the quasi-static measurements are included; showing that the two techniques agree both qualitatively and quantitatively.

Investigations in the intermediate regime were possible with Waxy Corn Starch, due to its larger particle size (see Eq. 11 and Table 11). However, the operating conditions that could be applied were constrained by the powder flow behaviour, i.e. since it is more free-flowing (see Fig. 2), the hardness is reduced, and the indenters penetrate further at a given velocity. In Figure 7 a the 
hardness of the starch compressed to $14.89 \mathrm{kPa}$ achieved by dropping Nylon,

\subsection{Determination of the operating conditions}

The previous sections showed that the dynamic indentation method can be used to measure $H_{d}$, provided that the measurement is operated in the range of reliable dimensionless penetration depth. Therefore, choosing an appropriate indenter (material density and size) and/or the height of release for investigating the powder behaviour in each flow regime (frictional, intermediate or collisional) can be problematic. For this reason, it could be extremely useful to develop a criterion for choosing the operating variables in order to respect the constraints 
imposed by the indenter penetration depth.

The following analysis aims at determining a generalized correlation between the range of dynamic hardness measurements reported above and the dimensionless shear rate in order to assist the operator in the choice of the correct experimental conditions. The correlation has to include all the relevant features of the indentation process, such as the properties of both the bulk powder and the indenter, as well as the drop height. These relevant variables have been combined to provide the following relevant dimensionless groups controlling the dynamic indentation:

$$
\frac{\rho_{b}}{\rho_{i}} ; \frac{R_{c}}{d_{p}} ; \frac{h}{R i} ; \frac{\rho_{b} \dot{\gamma}^{2} d_{p}^{2}}{\rho_{b} g h} .
$$

The first group in (6) takes into account the physical properties of the materials that constitute the intruder and the powder. In particular the powder bulk density, $\rho_{b}$, depends also on the pre-consolidation level of the powder and its cohesion. The second and the third groups describe the geometry of the indentation volume and introduce in the analysis some relevant length scales of the system: the crater radius, $R_{c}$; the particle size, $d_{p}$; the penetration level, $h$; and the indenter size, $R_{i}$. The two geometrical groups however are correlated to each other and hence they will be simplified in the following analysis. The last group describes the dynamics of indentation. It is the ratio between the inertial and the hydrostatic stresses which are representative of the stress state in the powder during and before indentation respectively. The shear rate in this group, as well as in $\dot{\gamma}^{*}$, is expressed using the crater radius, $R_{c}$, as previously explained.

It was found that the product of the four groups elevated to an appropriate exponent (see Eq. 7) makes all the data collapse on a master curve if plotted against the dimensionless shear rate, $\dot{\gamma}^{*}$.

$$
\left(\frac{\rho_{b}}{\rho_{i}}\right)^{1 / 2} \cdot\left(\frac{R_{c}}{d_{p}}\right)^{1} \cdot\left(\frac{h}{R i}\right)^{3 / 2} \cdot\left(\frac{\rho_{b} \dot{\gamma}^{2} d_{p}^{2}}{\rho_{b} g h}\right)^{1} .
$$

Manipulation of the four groups using their fitted exponents leads to further simplifications of the geometrical and dynamic groups, resulting in the following 
simple expression:

$$
\left(\frac{\rho_{b}}{\rho_{i}}\right)^{1 / 2} \cdot h^{*}\left(2-h^{*}\right)^{1 / 2} \cdot\left(\dot{\gamma}^{*}\right)^{2} .
$$

This scaling leads the data points to collapse on a straight line if plotted as a function of $\dot{\gamma}^{*}$ in a bi-logarithmic plot, as Fig. 9 shows. In agreement with all the observations made in section 3.2, this line has a positive slope, therefore teristic of the material and affected by particle shape and inter-particle friction [18, 20, 24, 26. To determine $\mathrm{C}$, one needs the unconfined yield stress from shear cell measurements, and the hardness value from quasi-static indentation, 

Constraint Factor of the material is simply $C=H_{s} / Y$. Once the constraint factor is characterized, the unconfined yield stress can be estimated in every condition by applying the inverse: $Y=H_{s} / C$.

The constraint factor of $\mathrm{TiO}_{2}$ R104, $\mathrm{TiO}_{2}$ DT51, Waxy Corn Starch and $C \approx 3$, meaning that, at least in these conditions, they are comparable to rigidperfectly plastic ductile metals [11, 18. For the starch $C \approx 1.9$, so it deviates from the behaviour of continuum solids. As expected, for $\mathrm{TiO}_{2}$ DT51, it has not been possible to find a constraint factor; this powder has neither a range of reliable indentation, nor a constant constraint factor. This particular powder was indeed the only one to have a quasi-static hardness varying in the entire range of penetration depths explored in Fig. 57a.

In figure 11 the yield strength inferred from the dynamic hardness and the constraint factor, are represented. In quasi-static conditions, the results are as the powder is pre-consolidated at the same stress. For instance, from the shear cell measurements we had for $\mathrm{TiO}_{2} \mathrm{R} 104 \mathrm{Y}=16.91 \mathrm{kPa}$ and $\mathrm{Y}=24.19$ $k P a$ respectively for Major Principal Stresses of 16.38 and $24.47 \mathrm{kPa}$. From dynamic hardness instead we had $\mathrm{Y}=18.28 \pm 2.31 k P a$ for compression of 20 $k P a$. Also for Waxy Corn Starch and $\alpha$-Lactose mono-hydrate, Y can be well inferred from dynamic hardness measurements; in particular one should note that the yield strength increases with $\dot{\gamma}^{*}$ in the intermediate regime of flow.

In conclusion, the dynamic indentation method is not only capable of capturing the dynamics of powders, but it also allows, if the constraint factor is

\section{Conclusions}

The objective of this work was to experimentally investigate the rheological behaviour of bulk solids in the intermediate regime of flow by relating the 
hardness to the shear rate.

Four bulk powders $\left(\mathrm{TiO}_{2}\right.$ R104, $\mathrm{TiO}_{2}$ DT51 $\alpha$-Lactose mono-hydrate and Waxy Corn Starch) were characterised at high shear rates by using a dynamic indentation process. The hardness results, evaluated in a range of dimensionless shear rates, confirm the trend observed by Pasha et al. [5]. Hardness does not vary with the shear rate in the quasi-static regime of flow, since friction between particles is predominant, an increase in hardness with the shear rate is observed in the intermediate regime of flow, where inter-particle interactions become more energetic [9]. However, the threshold dimensionless shear rate from which hardness becomes shear rate dependent cannot be universally defined. The reason is that the threshold shear rate is slightly dependent on the inter-particle cohesion; it increases if the powder cohesiveness increases [5].

However, the measurements show that indentation can well characterize the dynamics of powder from the quasi-static to the intermediate regimes. Furthermore, the boundary between these regimes correlates well with the findings of Tardos et al. 9. In order to explore the dynamic regime using indentation, greater values of dimensionless shear rate are required. However, increased shear rate results in greater penetration depths, which may extend beyond the operational window of indentation. Possible solutions to explore this regime are to use cohesive powders comprising larger particles (e.g. glass beads made cohesive by silanisation), or to use smaller or lighter indenters. However, for predicting 370 the best operating conditions one could use the proposed generalized relationship. Simulating by Discrete Element Method is a second possible alternative both for overcoming the difficulties in handling powders and for deepening the underlying theory of indentation.

The results, which are consistent with quasi-static indentation, verify the 375 possibility to use a dynamic ball indentation process for characterizing the flow resistance of powder beds. However, the method showed both strengths and weaknesses. The main advantage is that it allows the stress sensitivity of bulk solids to be investigated when subjected to shear rates that cannot be achieved with the common techniques (typically with a small powder quantity and low 

attachment by Freeman Technology.

\section{References}

[1] M. Leturia, M. Benali, S. Lagarde, I. Ronga, K. Saleh, Characterization of flow properties of cohesive powders: A comparative study of traditional

[4] H. M. Jaeger, S. R. Nagel, Physics of the granular state, Science 255 (5051) (1992) 1523-1531.

[5] M. Pasha, C. Hare, A. Hassanpour, M. Ghadiri, Numerical analysis of strain rate sensitivity in ball indentation on cohesive powder beds, Chemical and new testing methods, Powder Technology 253 (2014) 406-423.

[2] J. F. Klausner, D. Chen, R. Mei, Experimental investigation of cohesive powder rheology, Powder Technology 112 (1-2) (2000) 94-101.

[3] J. P. Seville, C.-Y. Wu, Particle Technology and Engineering: An Engineer's Guide to Particles and Powders: Fundamentals and Computational Approaches, Butterworth-Heinemann, 2016. Engineering Science 123 (2015) 92-98. 
[6] H. Salehi, D. Barletta, M. Poletto, D. Schütz, R. Romirer, On the use of a powder rheometer to characterize the powder flowability at low consolidation with torque resistances, AIChE Journal 63 (11) (2017) 4788-4798.

[7] A. Santomaso, P. Lazzaro, P. Canu, Powder flowability and density ratios: the impact of granules packing, Chemical Engineering Science 58 (13) (2003) 2857-2874.

[8] D. Schulze, Powders and bulk solids, Behaviour, Characterization, Storage and Flow. Springer (2008) 35-74.

[9] G. I. Tardos, S. McNamara, I. Talu, Slow and intermediate flow of a frictional bulk powder in the couette geometry, Powder Technology 131 (1) (2003) 23-39.

[10] C. Hare, U. Zafar, M. Ghadiri, T. Freeman, J. Clayton, M. Murtagh, Analysis of the dynamics of the ft 4 powder rheometer, Powder Technology 285 (2015) 123-127.

${ }_{420}$ [11] D. Tabor, The hardness of metals, Oxford university press, 2000.

[12] A. G. EVans, E. A. Charles, Fracture toughness determinations by indentation, Journal of the American Ceramic society 59 (7-8) (1976) 371-372.

[13] S. Ghosh, M. Tarafder, S. Sivaprasad, S. Tarafder, Experimental and numerical study of ball indentation for evaluation of mechanical properties and fracture toughness of structural steel, Transactions of the Indian Institute of Metals 63 (2-3) (2010) 617-622.

[14] A. M. Walsh, K. E. Holloway, P. Habdas, J. R. de Bruyn, Morphology and scaling of impact craters in granular media, Physical review letters 91 (10) (2003) 104301.

${ }_{430}$ [15] J. Simon, J. R. de Bruyn, Shape of impact craters in granular media, Physical Review E 76 (4) (2007) 041306. 
[16] F. Pacheco-Vázquez, J. Ruiz-Suárez, Impact craters in granular media: grains against grains, Physical review letters 107 (21) (2011) 218001.

[17] Y. Matsuda, S. Fukui, R. Kamiya, H. Yamaguchi, T. Niimi, Impact cratering on a granular bed by hydrogel spheres having intermediate property between solid and liquid, Physical Review E 99 (3) (2019) 032906.

[18] A. Hassanpour, M. Ghadiri, Characterisation of flowability of loosely compacted cohesive powders by indentation, Particle \& Particle Systems Characterization 24 (2) (2007) 117-123.

[19] J. Gubicza, A. Juhász, J. Lendvai, A new method for hardness determination from depth sensing indentation tests, Journal of materials research 11 (12) (1996) 2964-2967.

[20] M. Pasha, C. Hare, A. Hassanpour, M. Ghadiri, Analysis of ball indentation on cohesive powder beds using distinct element modelling, Powder technology 233 (2013) 80-90.

[21] M. Tirapelle, A. Santomaso, C. Hare, Characterising powder flowability at high shear rates by the ball indentation method, Chemical Engineering Transactions 74 (2019) 391-396.

[22] A. Jenike, Storage and flow of solids. university of utah engineering experiment station, Bulletin 123.

[23] Y. Tirupataiah, G. Sundararajan, The volume of the crater formed by the impact of a ball against flat target materials - the effect of ball hardness and density, International Journal of Impact Engineering 9 (2) (1990) 237-246.

[24] U. Zafar, C. Hare, A. Hassanpour, M. Ghadiri, Ball indentation on powder beds for assessing powder flowability: Analysis of operation window, Powder Technology 310 (2017) 300-306.

[25] W. Kang, Y. Feng, C. Liu, R. Blumenfeld, Archimedes' law explains penetration of solids into granular media, Nature communications 9 (1) (2018) 1101. 
[26] A. G. Stavrou, C. Hare, A. Hassanpour, C. Y. Wu, Investigation of powder flowability at low stresses by dem modelling, Chemical Engineering Science. In press. 


\section{List of Figures}

465

1 SEM micrographs of a) $\mathrm{TiO}_{2}$ R104, b) $\mathrm{TiO}_{2}$ DT51, c) $\alpha$-Lactose monohydrate and d) waxy Corn Starch. . . . . . . . . . 21

2 Flow functions and classification of the flow behaviour according

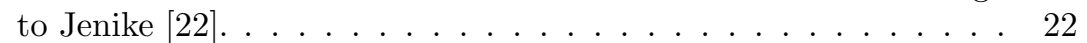

$3 \quad$ Range of reliable penetration depth. . . . . . . . . . . . 23

$4 \quad$ Experimental set-up. . . . . . . . . . . . . . . . . . . . . . 24

470

5 Quasi-static hardness of (a) $\mathrm{TiO}_{2}$ R-104, $\mathrm{TiO}_{2}$ DT51, (b) Waxy

Corn Starch and $\alpha$-Lactose mono-hydrate as a function of the dimensionless penetration depth. . . . . . . . . . . . . 25

1 6 Hardness of $\mathrm{TiO}_{2}$ R104 pre-consolidated at $20 \mathrm{kPa}$ (a) Vs di-

475

mensionless shear rate, $\dot{\gamma}^{*}$, and (b) Vs dimensionless penetration depth. In (b), the Dynamic ball indentation is compared with quasi-static hardness results. . . . . . . . . . . . . 26

7 Hardness of Waxy Corn Starch pre-consolidated at $14.89 \mathrm{kPa}$ (a) Vs dimensionless shear rate, $\dot{\gamma}^{*}$, and (b) Vs dimensionless \begin{tabular}{l|cccc} 
penetration depth. & . . . . . . . . . . . . . . . . . . . . . . . . . . & 27 \\
\hline Hardness of $\alpha$-Lactose mono-hydrate pre-consolidated at 5.75 &
\end{tabular}

480

1.
$k P a(\mathrm{a})$ Vs dimensionless shear rate, $\dot{\gamma}^{*}$, and (b) Vs dimensionless

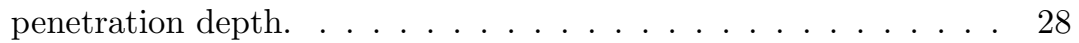

19 9 the dimensionless number in Eq. 8 | calculated for all the dynamic

485

hardness measurements is represented as a function of the dimensionless shear rate in a bi-logarithmic graph. All the observations

are fitted by linear regression. . . . . . . . . . . . . . . . 29

10 Materials constraint factor. . . . . . . . . . . . . . . . 30

11 Yield strength inferred by dynamic hardness measurements as a function of the dimensionless shear rate. Shear cell measure-

490

ments done at the same compaction level gave unconfined yield strengths equal to: $20.17 \mathrm{kPa}$ for $\mathrm{TiO}_{2} \mathrm{R} 104,1.97$ for waxy Corn Starch, and $1.98 \mathrm{kPa}$ in the case of Lactose mono-hydrate. . . . . 31 


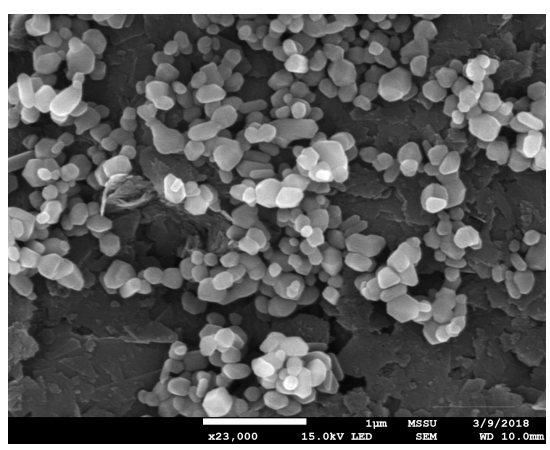

(a)

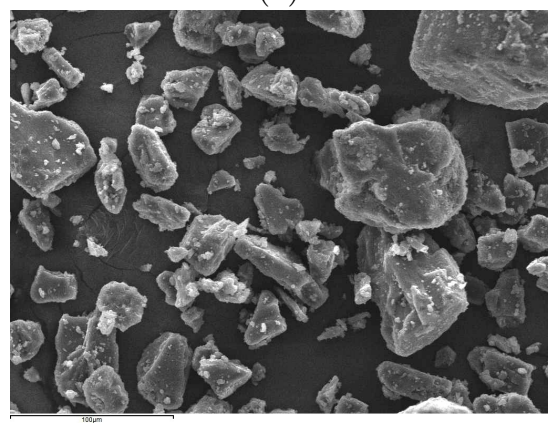

(c)

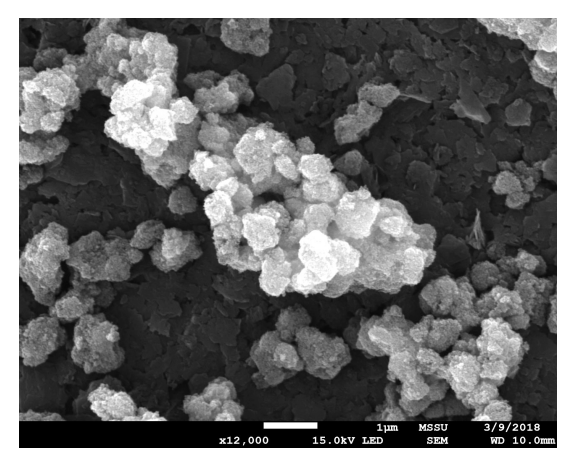

(b)

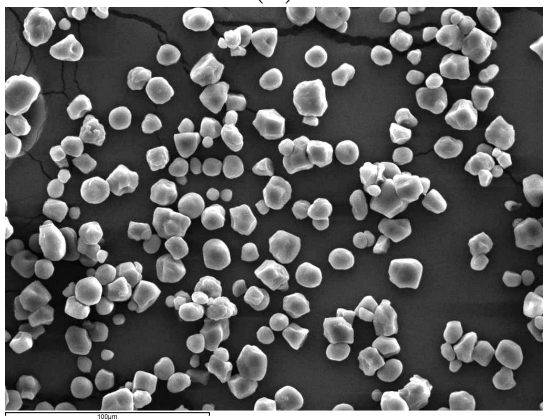

(d)

Figure 1: SEM micrographs of a) $\mathrm{TiO}_{2} \mathrm{R} 104$, b) $\mathrm{TiO}_{2}$ DT51, c) $\alpha$-Lactose monohydrate and d) waxy Corn Starch. 


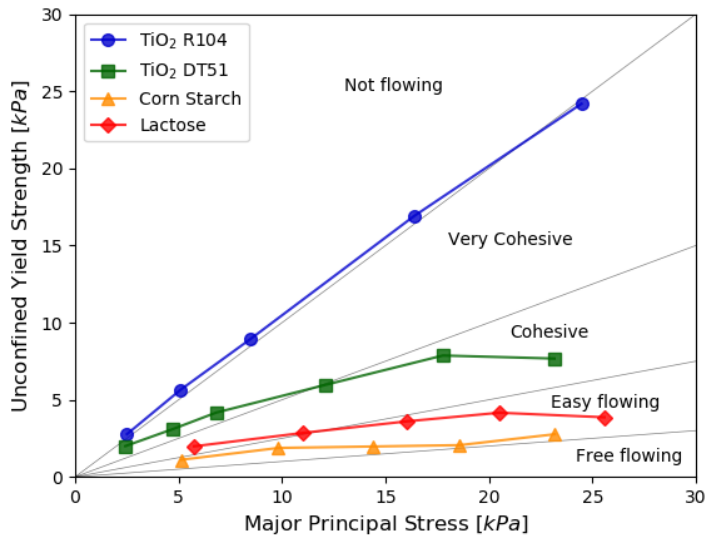

Figure 2: Flow functions and classification of the flow behaviour according to Jenike 22]. 


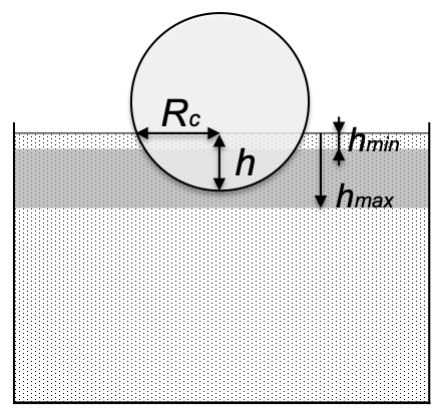

Figure 3: Range of reliable penetration depth. 


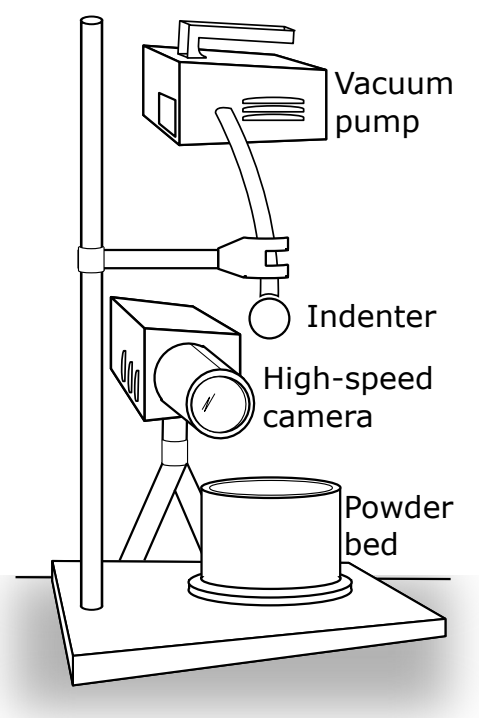

Figure 4: Experimental set-up. 


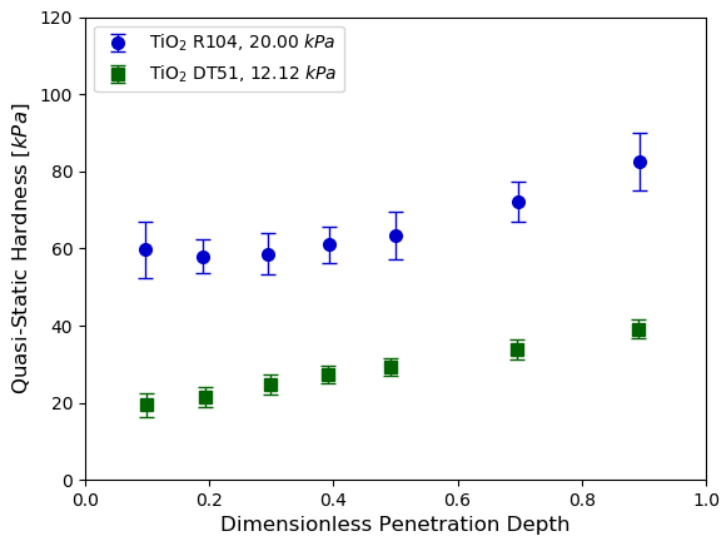

(a)

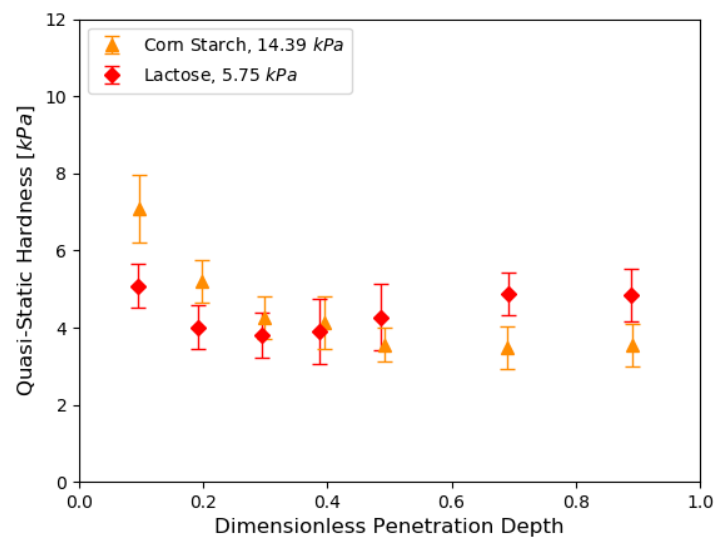

(b)

Figure 5: Quasi-static hardness of (a) $\mathrm{TiO}_{2}$ R-104, $\mathrm{TiO}_{2}$ DT51, (b) Waxy Corn Starch and $\alpha$-Lactose mono-hydrate as a function of the dimensionless penetration depth. 


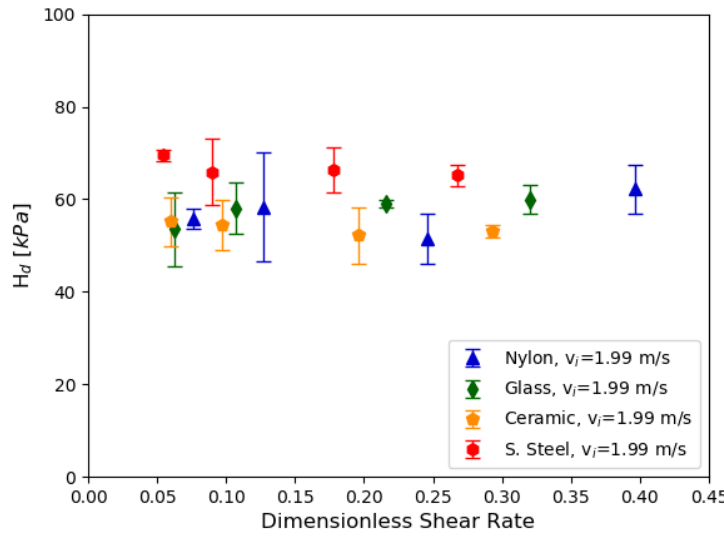

(a)

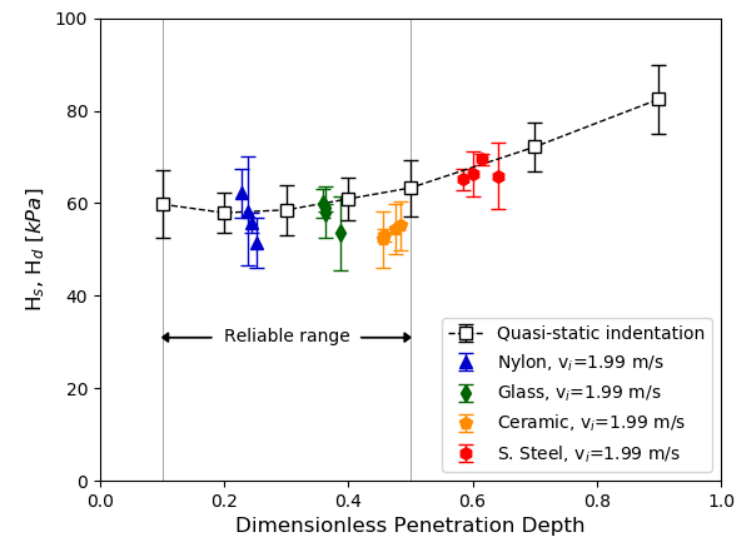

(b)

Figure 6: Hardness of $\mathrm{TiO}_{2} \mathrm{R} 104$ pre-consolidated at $20 \mathrm{kPa}$ (a) Vs dimensionless shear rate, $\dot{\gamma}^{*}$, and (b) Vs dimensionless penetration depth. In (b), the Dynamic ball indentation is compared with quasi-static hardness results. 


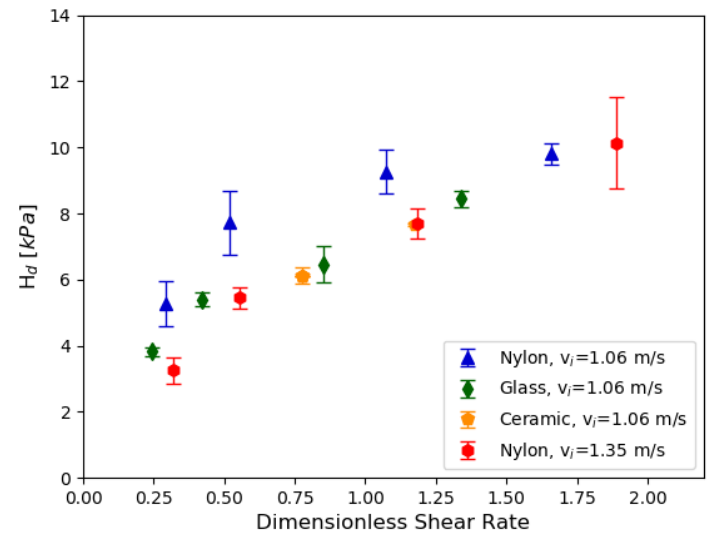

(a)

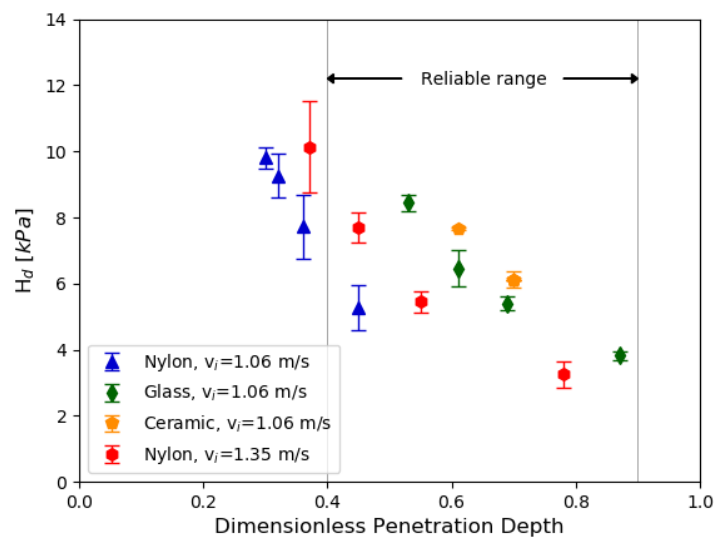

(b)

Figure 7: Hardness of Waxy Corn Starch pre-consolidated at $14.89 k P a$ (a) Vs dimensionless shear rate, $\dot{\gamma}^{*}$, and (b) Vs dimensionless penetration depth. 


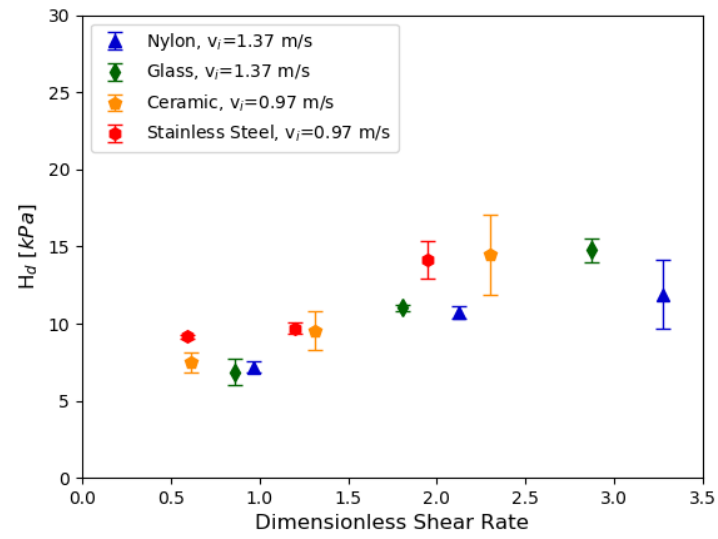

(a)

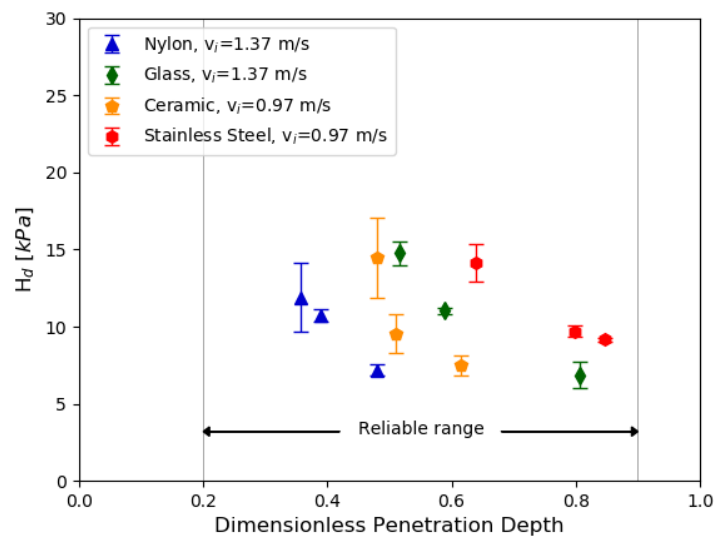

(b)

Figure 8: Hardness of $\alpha$-Lactose mono-hydrate pre-consolidated at $5.75 \mathrm{kPa}$ (a) Vs dimensionless shear rate, $\dot{\gamma}^{*}$, and (b) Vs dimensionless penetration depth. 


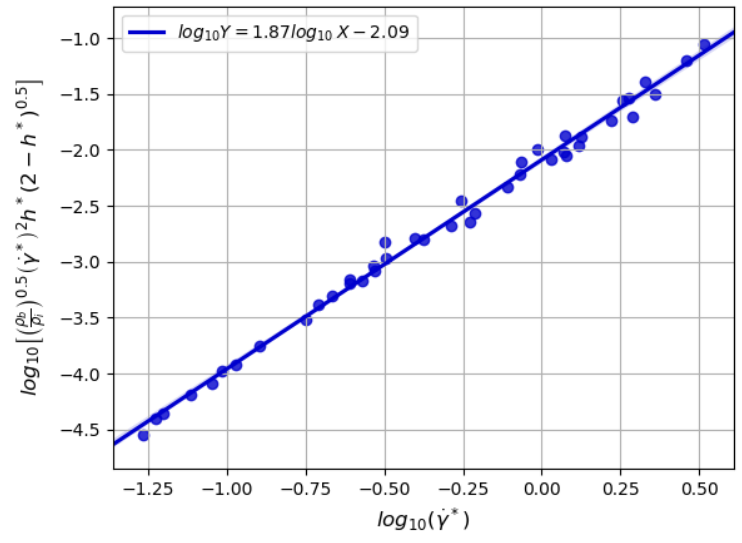

Figure 9: the dimensionless number in Eq. 8 calculated for all the dynamic hardness measurements is represented as a function of the dimensionless shear rate in a bi-logarithmic graph. All the observations are fitted by linear regression. 


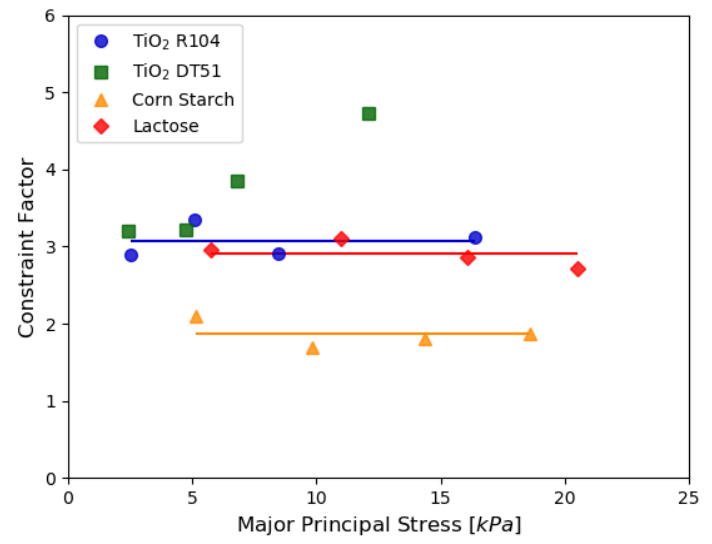

Figure 10: Materials constraint factor. 


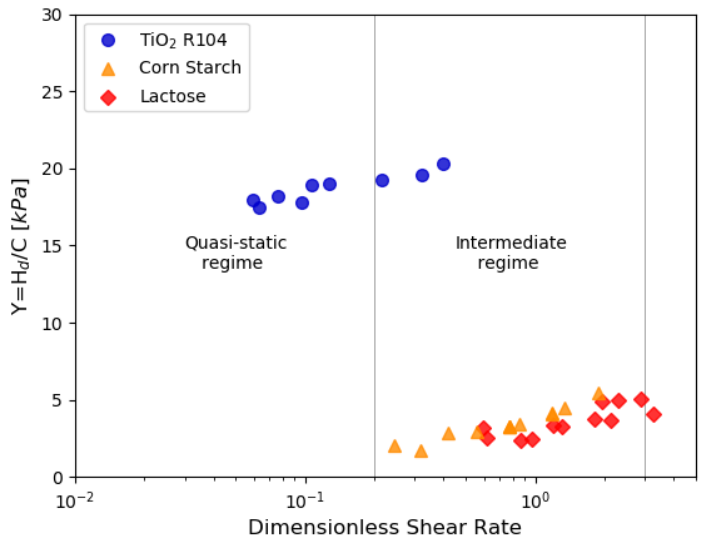

Figure 11: Yield strength inferred by dynamic hardness measurements as a function of the dimensionless shear rate. Shear cell measurements done at the same compaction level gave unconfined yield strengths equal to: $20.17 \mathrm{kPa}$ for $\mathrm{TiO}_{2}$ R104, 1.97 for waxy Corn Starch, and $1.98 \mathrm{kPa}$ in the case of Lactose mono-hydrate. 


\section{List of Tables}

1 Materials equivalent volumetric diameters and flow indices at dif-

495 ferent levels of pre-shear normal stress. . . . . . . . . . . 33 
Table 1: Materials equivalent volumetric diameters and flow indices at different levels of pre-shear normal stress.

\begin{tabular}{lccccccccccc}
\multirow{2}{*}{ Material } & \multicolumn{4}{c}{ Equivalent diameters $[\mu m]$} & \multicolumn{1}{c}{ Pre-shear $[k P a]$} \\
\cline { 2 - 13 } & $d_{10,3}$ & $d_{50,3}$ & $d_{90,3}$ & $d_{99,3}$ & 1 & 2 & 3 & 6 & 9 & 12 & 15 \\
\hline $\mathrm{TiO}_{2}$ R104 & 0.22 & 0.38 & 0.74 & 1.04 & 0.90 & 0.90 & 0.95 & 0.97 & 1.01 & - & - \\
$\mathrm{TiO}_{2}$ DT51 & 0.71 & 1.08 & 1.68 & 2.08 & 1.22 & 1.53 & 1.64 & 2.03 & 2.27 & 3.05 & - \\
$\alpha$-Lactose & 34.67 & 82.80 & 157.09 & 233.87 & - & - & 2.90 & 3.86 & 4.48 & 4.93 & 6.65 \\
Corn Starch & 13.82 & 30.60 & 65.20 & 150.00 & - & - & 4.64 & 5.28 & 7.31 & 9.08 & 8.46 \\
\hline
\end{tabular}

